ORIGINAL RESEARCH PAPER

\title{
STUDY OF STRUCTURAL-MECHANICAL CHARACTERISTICS OF EMULSION-FOAM SYSTEMS OF MILK CREAM AND HYDROCOLOIDS
}

\author{
YULIIA KAMBULOVA ${ }^{1}$, YULIIA ZVIAHINTSEVA-SEMENETS ${ }^{1}$, ANASTASIIA \\ SHEVCHENKO $^{2 *}$, OLENA KOKHAN ${ }^{1}$ \\ ${ }^{1}$ Department of Technology of Bakery and Confectionary Goods, Educational and Scientific Institute of Food \\ Technologies, National University of Food Technologies, 68 Volodymyrska street, 01601 Kyiv, Ukraine \\ ${ }^{2}$ Problem Scientific and Research Laboratory, National University of Food Technologies, 68 Volodymyrska street, \\ 01601 Kyiv, Ukraine \\ *Corresponding author:nastyusha8@ukr.net
}

Received on 1 April 2020

Revised on 6 October 2020

\begin{abstract}
The current trends in food production are aimed at creating products with reduced energy value. The present study investigates the emulsion-foam structures of the cream from churned cream with a $20 \%$ fat content used in decorating flour confectionery. It is proposed to stabilise such systems by the introduction of sodium alginate, 1 -carrageenan or gelatin. It was determined that the emulsionfoam structures of the cream samples under investigation belong to the class of pseudoplastic non-Newtonian fluids. The different values of the conditional dynamic limit of fluidity were determined: for sodium alginate $\sim 100 \mathrm{~Pa}$, for $1-$ carrageenan $\sim 35 \mathrm{~Pa}$, for gelatin $-66 \mathrm{~Pa}$, which is connected to a high level of swelling of the hydrocolloids in cream and their differences in structure-forming manners. Within 2 hours upon obtaining, all samples are characterized by a decrease in the total deformation of the sample. For the samples under study, plastic deformations exceeded springy deformations. In the structure of the experimental samples, plastic deformations exceeded springy deformations: the relative plastic deformation of the samples of emulsion-foam systems after churning was $98 \ldots 99 \%$. Over time a part of the springy deformation increased for the samples with gelatin and sodium alginate, and decreased for the samples with i-carrageenan.
\end{abstract}

Keywords: emulsion-foam systems, milk cream, hydrocolloids, gelatin, sodium alginate, 1 -carrageenan, rheological properties, springy-plastic properties

\section{Introduction}

Recently, more and more food products based on emulsion-foam systems, have been created. Due to the recommendations of the World Health Organization on the correction of the quantity and quality of fats entering the human body, namely

https://doi.org/10.35219/foodtechnology.2020.2.06 
that the total consumption of fats should not exceed $30 \%$ of all energy, and industrial trans-fats should be excluded, the tendency is to turn to the development of low fat natural cream systems.

A lot of confectionery and desserts are made using emulsion-foam systems, obtained by churning milk cream (Zaouadi et al., 2019; Barata et al., 2019; Kaledina et al.,2017; AlAmin., 2019; Hartel et al.,2018; Schmidt et al., 2016; Goff et al., 2001) with a fat content of at least $35 \%$ with sugar. The quality of such a system depends on the maturation of the cream that is necessary to obtain a denser and foamy structure and low churning temperature $\left(0 \ldots 6^{\circ} \mathrm{C}\right)$, which provides foam stability.

The process of cream foaming is based on the formation of a layer of protein-fat globules around the bubbles of air that is dispersed in the system during churning (Dewettinck et al., 2008, Nguyen et al, 2015). The initial churning stage involves the stabilization of the trapped air bubbles by a temporary interfacial film of soluble whey proteins and $\beta$-casein. The protein globules adsorbed on the surface of the bubbles are thought to denature, leading to a viscous-springy membrane formation around the air phase, which protects the emulsion from coalescence (Schamm, 2006). Protein adsorption plays an important role in the capture of air bubbles, but structure formation occurs due to the binding interactions between the fat droplets and the air bubbles.

During churning, the fat globules lose segments of their natural membrane, thereby exposing the hydrophobic surfaces of pure fat. There is partial coalescence of fat droplets (destabilization). Gradually, these partially destabilized fat globules are adsorbed around the air bubbles, forming a rigid framework. The fat frame formed provides stability and texture for the final product.

The strength of the obtained foam depends on the size of the fat frame - the larger it is, the more resistant the foam is. Therefore, reducing the fat content in emulsion foam systems always requires the introduction of additional stabilizing additives, usually hydrocolloids or substances containing natural hydrocolloids (Milliatti et al., 2018, Yousefia et al., 2019, Javidi et al., 2019, Farahmandfar et al., 2019). Many stabilization methods suggest the use of gelatin (Duan et al., 2018, Rahimah et al., 2020, Lysyuk et al., 2007). However, the system with gelatin loses its plasticity, acquires signs of jelly-like structure, and has a higher density. Another trend is related to the use of fruit and vegetable raw materials as a source of pectin substances and fibre. For example, researchers (Melnychuk et al., 2011) developed a method of obtaining an emulsion-foam system of cream from milk cream with a fat content of $30 \%$ with the addition of hydrolyzed carrot or pumpkin puree. The cream, which contains $0.7-1 \%$ pectin, increased the stability of the cream over time. Ershova et al., (2008) developed the technology of production and assortment of low-calorie functional cream based on blending vegetable oils and a vegetable emulsifier - the root of Aralia amanduria (Araiiamandshurica Rupr. Et Maxim) and the root of licorice (Glycyrrnizaglabra L.). The developed assortment additionally includes fruit and berry and vegetable purees in the recipe, which allow the formation of colour, taste and aromatic properties of creams without artificial dyes and flavours. The use of vegetable oils in the cream provides consumer benefits (for example, reducing cholesterol, 
providing a high level of essential fats, reducing acidity), while cutting the cost of raw materials as compared to milk fat. However, problems arise when structuring and stabilizing the structures of the final product. Mureșan et al. (2018) studied the possibility of improving the consumer and functional properties of recombinant churned creams by developing model systems for food fats. These models contained anhydrous milk fat mixed with different amounts $(15 \%, 30 \%, 45 \%)$ of oleogel from rice bran.

Radzievska et al. (2013) substantiated and developed the technology of low-calorie emulsion confectionery cream based on vegetable cream. The cream contained refined deodorized sunflower oil, an aqueous solution of native potato starch, an emulsifier E471 (mono- and diglycerides of fatty acids), sugar and cocoa powder. It was experimentally established that the mass fraction of oil in the recipe of the cream should be $25 \%$; under such conditions there was no separation of water and delamination of the emulsion, and the mass retained homogeneity.

In another study, the possibility of stabilizing the structure of the vegetable cream with a fat content of $25 \%$ by introducing agar, pectin and gelatin was investigated (Romanova et al., 2008). The rational concentrations of jelly-forming agents were: $0.36-0.67 \%$ of agar; pectin $-0.44-1.02 \%$; gelatin $-1.9-2.0 \%$. In Srikaeo et al. (2020), the effect of pectin and carrageenan on the physical properties and lipid digestion of churned cream was evaluated, and hydrocolloids were shown to improve foaming properties and delay the rate of lipid digestion.

Gurov (2007) improved the technology of low-calorie cream-churned semi-finished emulsion-foam structure, which was stored in the form of liquid water-fat emulsion, and churned when cooked. The product contained sodium caseinate, sodium alginate, carboxymethylcellulose and vegetable fat. Based on the semi-finished product, scientists proposed to develop creams with an emulsion-foam structure that meet the requirements of healthy nutrition in terms of quality.

The technology of obtaining low-calorie churned semi-finished products (Lingerud, 2009), which have high functional properties after churning and a pleasant aroma was proposed. The fat phase consisted of $10 \ldots 25 \%$ fat, $5 \ldots 60 \%$ unsaturated fatty acids, and the rest was lactic fat.

The low-calorie emulsion-foam cream system proposed by Allen et al. (2005) included water, coconut oil, hydrolyzed corn starch, powdered milk, whey protein, and oil flavouring.

The information above confirms the interest of food industry professionals in reducing the energy value of emulsion-foam systems as a basis for cream products. Scientists are developing technologies aimed at regulating the fat phase, improving the process and the formulation of the finished product in accordance with the concepts of modern nutrition. A large part of the development is devoted to improving the technology of semi-finished products based on cream of vegetable origin, the creation of a blending basis for further churning (powdered milk with the addition of milk protein (sodium caseinate), vegetable fats and industrial fats, emulsifiers, stabilizers. However, the use of natural milk fats in technology is more necessary in terms of the benefits to the human 
body, and the issues of ensuring the stable quality of the emulsion-foam structure of creams with reduced fat content need to be studied.

The purpose of this study was to determine the structural and mechanical properties of emulsion-foam systems consisting of churned cream with a fat content of $20 \%$, stabilized with i-carrageenan or sodium alginate.

The structural-mechanical properties of emulsion-foam systems were studied in point of rheological parameters and springy-plastic deformation.

It can be stated that the rheological properties of emulsion-foam systems will depend on the interaction with the rheology of the dispersion medium and the characteristics of the suspended particles. In emulsion-foam systems, the rheology of the dispersion medium usually plays a major role in the overall rheology of the system. Gel-formers available in the dispersion medium develop a solid net over time that provides stable indicators of the emulsion-foam stability. Accordingly, they change the nature of the dispersion medium, affect the process of milk fat destabilization, thereby changing the rheology of both the dispersion medium and the system as a whole.

The volume fraction of the dispersed phase (air) will also be influenced. The viscosity of the emulsion will increase with the increase in the volume fraction of the dispersed phase, due to the energy dissipation (scattering) associated with the fluid flow.

The reason of dissipation can be the thermal motion of molecules: when the selected volume of matter moves, the molecules at the volume boundary, moving chaotically, are constantly in contact with the molecules of other volumes, resulting in a continuous exchange of momentum and matter between the volumes of the medium.

\section{Materials and methods}

The following raw materials were used to make emulsion-foam systems for pastry cream from churned cream: milk cream with a fat content of $20 \%$; white crystalline sugar (sucrose); food gelatin; sodium alginate, t-carrageenan ('GERoeperGmbH', Germany).

The composition of the experimental samples, $\mathrm{g} / 100 \mathrm{~g}$, and the physico-chemical parameters of the raw materials are shown in Table 1.

The churning of all the cream samples was carried out using a hand mixer Bosch MFQ4020 (Slovenia) with the speed of the whipping body of $300 \mathrm{rpm}$ for 15 minutes.

For the preparation of the emulsion-foam system with sodium alginate, the formulation amount of the structure-forming agent was mixed with sugar (sucrose) in a $1: 1$ ratio, introduced into the cream, heated to a temperature close to $85-90^{\circ} \mathrm{C}$ for complete dissolution, and then cooled to $8-12{ }^{\circ} \mathrm{C}$. The cooled solution was added to the main part of the cream and churned with the gradual addition of the remaining sugar (sucrose). 
Table 1. Prescription composition of experimental samples, g / 100g,and physico-chemical parameters of raw materials.

\begin{tabular}{ccccc}
\hline \multirow{2}{*}{ Raw material } & \multicolumn{2}{l}{ Formulation of the obtained samples, g/100g } & \multirow{2}{*}{ Dry matter, \% } \\
\cline { 2 - 4 } & $\begin{array}{c}\text { Control } \\
\text { sample }\end{array}$ & Sample 1 & Sample 2 & \\
\hline cream with 20\% & 85.0 & 85.0 & 85.0 & 27.00 \\
fat & 20.0 & 20.0 & 20.0 & 30.00 \\
sugar & 2.0 & - & - & 89.85 \\
food gelatin & - & 0.72 & - & 88.00 \\
sodium alginate & - & - & 0.72 & 90.00 \\
1-carrageenan & - & & & \\
\hline
\end{tabular}

For the preparation of creams with 1 -carrageenan, the structure-forming agent was mixed with all the recipe amount of sugar (sucrose), diluted with cream, heated to complete dissolution in the temperature range of $90-95{ }^{\circ} \mathrm{C}$ and cooled to a temperature of $6-10^{\circ} \mathrm{C}$, after which the cooled solution was introduced while churning the main part of cream.

The control sample was the formulation of the emulsion-foam structure of the cream, for the preparation of which gelatin was soaked in the cream in a 1:10 ratio. The mixture was heated up to the complete dissolution of gelatin in the temperature range $60-65^{\circ} \mathrm{C}$, then cooled to a temperature of $40-45^{\circ} \mathrm{C}$. The cooled solution was introduced to the main part of the cream with the gradual addition of the remaining sugar.

The rheological properties of cream samples were determined on a rotational viscometer Rheotest RN 4.1 (Germany) according to the methods presented by Erkebaev et al. (2006), Kovalevska (2010), Mezger (2006) and Volovik et al. (1999). The shear stress was calculated by the following formula:

$$
\tau=\mathrm{Z} \times \alpha
$$

where: $\tau$ is the shear stress; $\mathrm{Z}$ is the measurement steam constant (for the moving cylinder $\mathrm{H} Z=2.89$ ); $\alpha$ is a value on the scale of the recording device.

The maximum viscosity, $\eta_{\max },(\mathrm{Pa} \cdot \mathrm{s})$ and the minimum viscosity, $\eta_{\min },(\mathrm{Pa} \cdot \mathrm{s})$ were calculated by the formula:

$$
\eta=\frac{\tau}{\dot{\gamma}}
$$

where: $\gamma$ is shear rate gradient, $\mathrm{s}^{-1}(0.3333 ; 0.6 ; 1 ; 1.8 ; 3 ; 5.4 ; 9 ; 16.2 ; 27.6 ; 48.6$; $81 ; 145.8)$.

Based on the obtained results, the rheological viscosity $\eta=f(\gamma)$ and flow curves $\tau$ $=\mathrm{f}(\gamma)$ were constructed (Figure 1); using the flow curves, a number of rheological parameters were determined, namely the conditional static yield stress $\mathrm{P}_{\mathrm{k} 1}$, which corresponds to the beginning of the deformation of the sample, the conditional 
dynamic yield stress $P_{k 2}$, above which the deformation rate increases significantly, and the shear stress corresponding to a completely destroyed structure, $\mathrm{P}_{\mathrm{m}}, \mathrm{Pa}$.

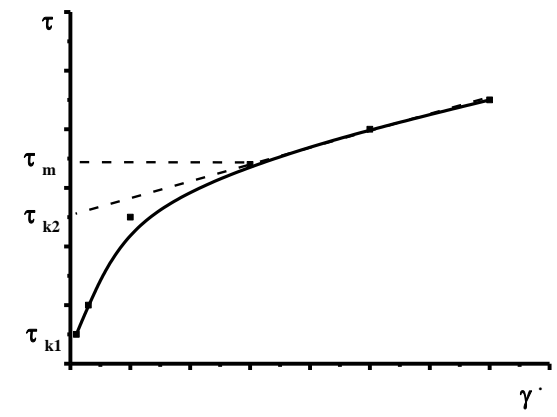

a

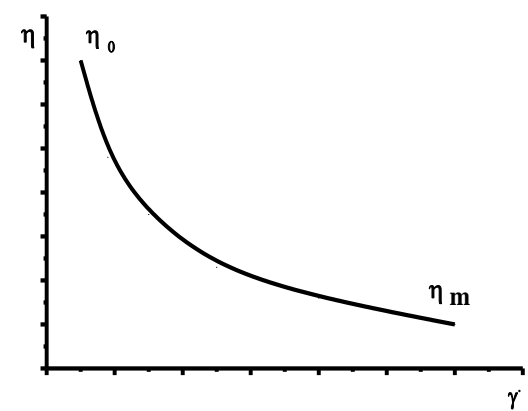

b

Figure 1. Rheological flow (a) and (b) viscosity curves.

The strength of the supramolecular structure formed in the system was considered as the value obtained from the difference between the values of the maximum (minimum shear gradient) and minimum (maximum shear gradient) viscosity ( $\eta_{\max }$ $\left.-\eta_{\min }, \mathrm{Pa} \cdot \mathrm{s}\right)$.

The conditional static yield stress, $\mathrm{P}_{\mathrm{k} 1}$ is defined as the shear stress with a minimum shear gradient.

The conditional dynamic yield stress, $\mathrm{P}_{\mathrm{k} 2}$, is defined as the point of intersection of the sample tangent and the yield curve of sample, constructed using at least three values of the shear gradient at which the fluidity of the samples is observed.

The following ratios were considered in order to further characterize the deformation behaviour of the system: $\mathrm{P}_{\mathrm{k} 1} / \mathrm{P}_{\mathrm{k} 2}$ the strength of structural connections the greater this ratio, the stronger the structural connections formed in the system; $\mathrm{P}_{\mathrm{m}} / \mathrm{P}_{\mathrm{k} 1}$ - the destruction of the structure - characterizes the range of stresses in which the destruction of the structure takes place.

The determination of the rheological parameters of emulsion-foam creams systems was carried out at $2 \ldots 4^{\circ} \mathrm{C}$ immediately after obtaining them $(0 \mathrm{~min})$, then after 30 , 60 and $120 \mathrm{~min}$.

The springy-plastic characteristics of the studied creams were determined by means of a ST-2 structrometer (LLC "Laboratory of Quality", Moscow), which provided information on the total deformation of the test samples, the development of springy or plastic deformation; the calculation of the conditional strength of the system; the specific work of plastic deformation and other structural and mechanical characteristics. A detailed description of the configuration of the structrometer is shown in Figure 2.

The operating principle of the structrometer is based on measuring the structural and mechanical characteristics of the test sample of a cylindrical form, $3 \mathrm{~cm}$ in diameter and $5 \mathrm{~cm}$ in height, located on a plate, under the action of load. 


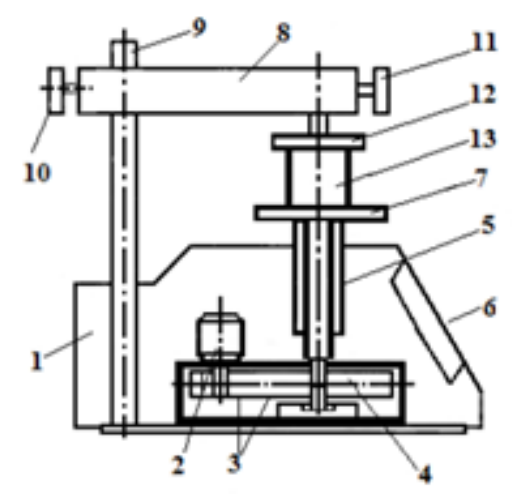

Figure 2. Scheme of the structrometer ST -2 .

1 -frame; 2 - motor; 3 - gears; 4 - screw; 5 - nut; 6 - control unit; 7 - plate; 8 - measuring head; 9 - barbell; 10 - the screw for fastening of a measuring head; 11 - the screw for fastening of a body of penetration; 12 - variable indenter; 13 - sample of the studied food product.

The main parts of the structrometer are the control unit (6) and the replaceable measuring heads (8). In the upper part of the control unit there is a round table (7) which moves vertically. Above the table there is a measuring head, which moves along the bar (9).

The technical characteristics of the device: range of speed of table movement - 1$100 \mathrm{~mm} / \mathrm{min}$; load measurement range - 0.1-100 N; load measurement error - no more than 1\%; displacement measuring range $0-43 \mathrm{~mm}$; relative error of time measurement - less than 1 (Levit et al.,2014).

Measurements were performed at $4-8{ }^{\circ} \mathrm{C}$, and the analysis was conducted immediately after obtaining the samples ( $0 \mathrm{~min})$, then after 30, 60, $120 \mathrm{~min}$, and following the formation dynamics of the structure development.

The investigation was carried out in the following conditions: touch force $\mathrm{F}_{0}=0.5$ $\mathrm{N}$; table speed $v=0.1 \mathrm{~m} / 60 \mathrm{~s}$; maximum load $\mathrm{F}=2 \mathrm{~N}$ per sample. The experimental data obtained were expressed as deformation kinetics curves (Figure 3) (Erkebaev et. al., 2006). According to the deformation curves, the total deformation of the sample $\left(\mathrm{H}_{1}, \mathrm{~m}\right)$, plastic deformation $\left(\mathrm{H}_{2}, \mathrm{~m}\right)$, and springy deformation $\left(\mathrm{H}_{3}, \mathrm{~m}\right)$ as the difference between the total deformation of the sample, $\mathrm{H}_{1}$, and plastic deformation, $\mathrm{H}_{2}$, were determined and used to determine the following:

-the relative plastic deformation,

$\varepsilon_{\mathrm{pl}}$

$$
\mathcal{E}_{\mathrm{pl}}=\left(\frac{\mathrm{H}_{2}}{\mathrm{H}_{1}}\right) \times 100 \%
$$

- the relative springy deformation,

$\mathcal{E}_{\mathrm{sp}, \%}$ :

$$
\mathcal{E}_{\mathrm{sp}}=\left(\frac{\mathrm{H}_{3}}{\mathrm{H}_{1}}\right) \times 100 \%
$$


- the conditional strength limit, $\delta$, Pa:

$$
\delta=\frac{4 \mathrm{~F}}{\pi \mathrm{D}^{2}} \times 10^{6}
$$

- the specific work of plastic deformation, $\mathrm{J} / \mathrm{m}^{3}$ :

$$
A_{\text {spec }}=\frac{S \cdot M_{\mathrm{f}} \cdot M_{\mathrm{H}}}{1000 \cdot \mathrm{V}_{0}} \times 10^{6}
$$

where: $\mathrm{S}$ is the area under the curves (the shaded part of the figure), $\mathrm{m}^{2} ;{ }_{f}$ is the scale of the graph along the axis of the load force, $\mathrm{N} / \mathrm{m} ; M_{\mathrm{H}}$ is the scale of the figure along the axis of deformation, $\mathrm{m} / \mathrm{m} ; V_{0}$ is the sample volume, $\mathrm{m}^{3}$.

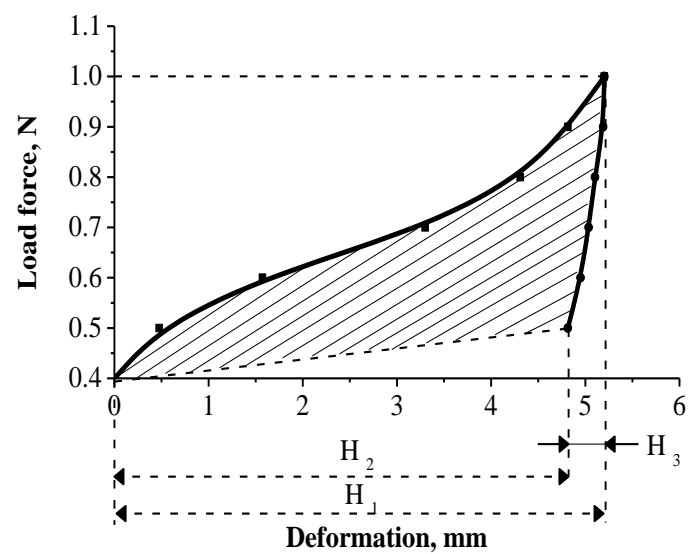

Figure 3. The curve of kinetics of the samples' deformation

\section{Statistical analysis}

The statistical processing of the result values was performed by sequential regression analysis using the Microsoft Excel XP and Origin Pro8 software.

\section{Results and discussion}

The viscosity and flow curves of the cream samples tested are shown in Figures 4-6, and the analysis of the results is included in Table 2.

As it can be seen from figures 4,5 and 6 , when increasing the shear stress, the system collapsed and acquired fluidity that is characteristic of non-Newtonian fluids as shear type or pseudoplastic non-Newtonian fluids (Chen et al., 2016, UrRehman et al., 2017).

It was determined that the structure of the creams under study became more stiff (higher viscosity) in time. For example, the value of the maximum viscosity for the gelatin cream over 120 minutes after obtaining increased by almost 44\%; for creams with 1 -carrageenan - by $37 \%$, for creams with sodium alginate - by $22 \%$. This can be due to the development of a gel-like structural framework of hydrocolloids, slowing the movement of macromolecules, and the final fixation of aggregates in the frame by volume of the dispersion medium. The formation of the 
hydrocolloids' gel-like structures in the cream was also reported in Camacho et al. (2005), and Farahmandfar et al. (2017).

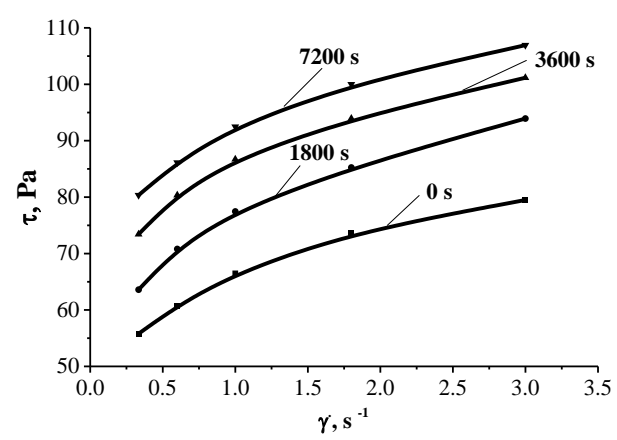

a

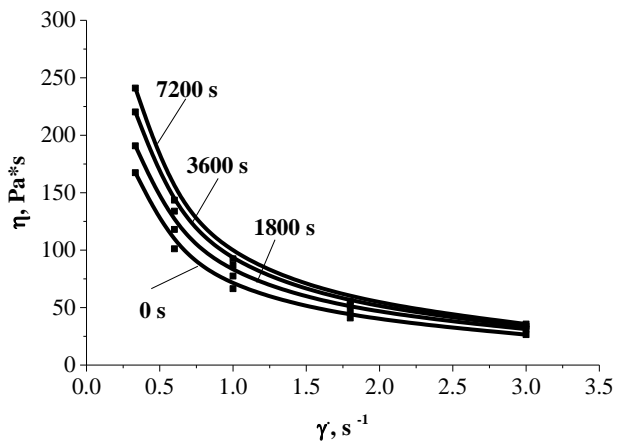

b

Figure 4. Rheological flow (a) and viscosity curves (b) of emulsion-foam cream system with gelatin and sucrose.

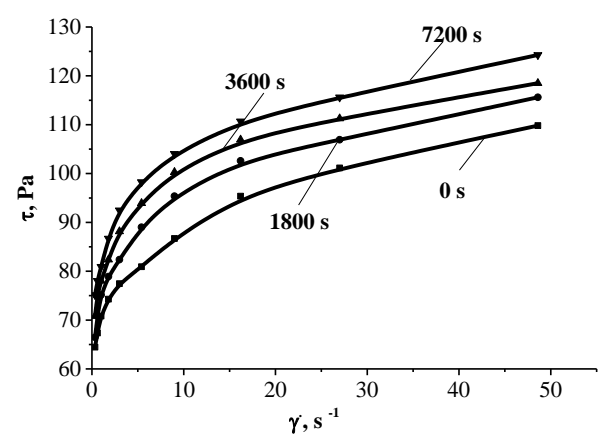

a

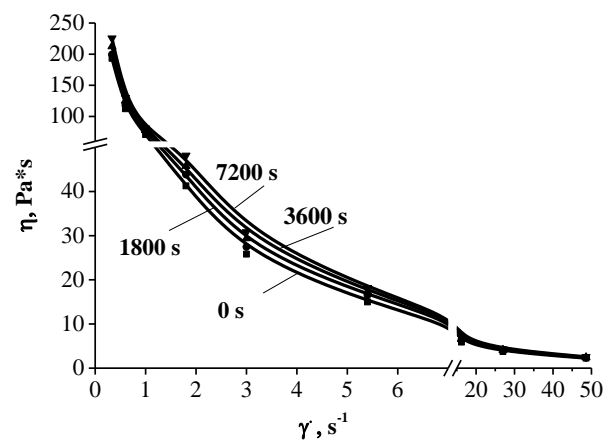

$\mathrm{b}$

Figure 5. Rheological flow (a) and viscosity curves (b) of emulsion-foam cream system with sodium alginate and sucrose.

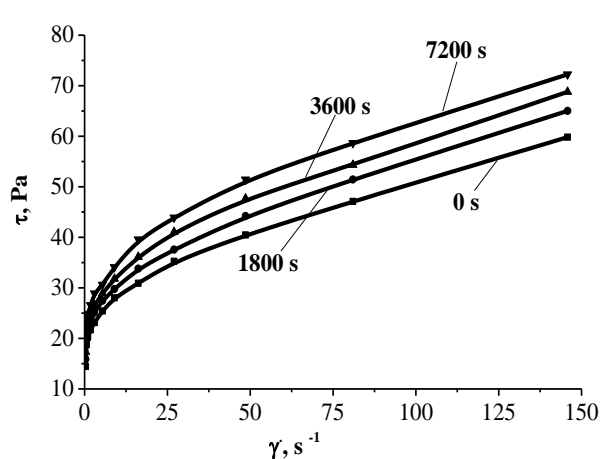

a

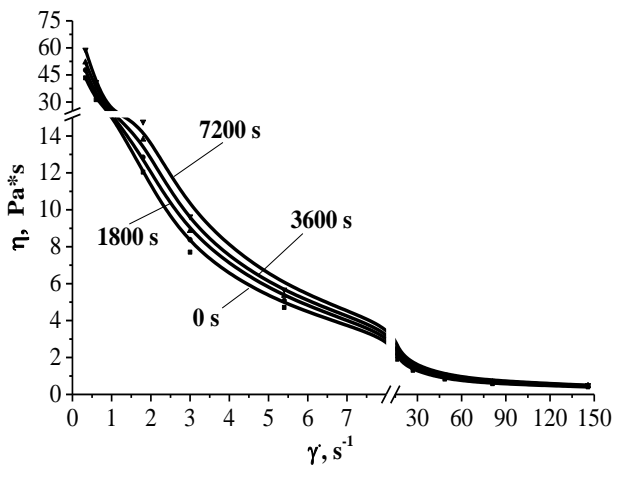

b

Figure 6. Rheological flow (a) and viscosity curves (b) of emulsion-foam cream system with i-carrageenan and sucrose.

Scientific studies demonstrated rheological properties (gelling and melting) and microstructural properties (typical casein network with thin filamentary structures) 
of gelatin gels that were similar to the xanthan gum / carob gum combination (Pang et al., 2016).

Among the maximum viscosity values registered for the emulsion-foam systems of the creams with different structure-forming agents - alginate, carrageenan and gelatin, there was a significant difference. In our opinion, it could be related to the different degree of swelling of hydrocolloids and the different speed of swelling. This process is due to the amount and reactivity of the hydrogen bonds. The process of swelling of sodium alginate and t-carrageenan in cream was studied in detail (Zviahintseva-Semenets et al., 2016) and it was found that 1-carrageenan differs from sodium alginate by a smaller degree of swelling at temperatures within the range $25 \ldots 100^{\circ} \mathrm{C}$. Accordingly, the degree of swelling of the sodium alginate was higher, which in our opinion reflects on the viscosity of the dispersed medium. The rheological indicators of pure colloidal solutions of gelatin, sodium alginate and t-carrageenan were further investigated (Kambulova et al., 2017), and it was confirmed that the higher viscosity values of virtually non-destructive systems were inherent to solutions with sodium alginate as compared to solutions with tcarrageenan and with gelatin.

The rheological parameters of the emulsion-foam systems of the cream in churned creams are shown in Table 2. The table data show that when increasing the rotational speed, all the systems manifested shear-thinning behaviour. For the samples containing polysaccharides, different values of the the dynamic limit of the system's ability to fluidity $\left(\mathrm{P}_{\mathrm{k} 2}\right)$ at the beginning of the flow were observed: for sodium alginate $\sim 100 \mathrm{~Pa}$ and more, for 1 -carrageenan $\sim 35 \mathrm{~Pa}$ and more. These mean that the structures with sodium alginate and 1-carrageenan will withstand the different intensity of mechanical loading. Such differences should be taken into account when decorating semi-finished baked products.

The differences in point of rheological parameters can be explained by the mechanism of gelation in dairy products. Sodium alginate forms a threedimensional gel grid characteristic to the calcium-alginate reaction mechanism. Associates are formed due to the occurrence of nodal zones at the physical junctions of the block of $\lambda$-L-guluronic acid of one sodium alginate molecule with the block of $\lambda$-L-guluronic acid of another molecule of sodium alginate by the "chain-Ca ${ }^{2+}$-chain" interaction (King, 2019, Jiao et al., 2019). In addition, dairy systems witness the formation of preservatives like $\beta$-lactoglobulin, serum albumin and sodium alginate, which contributes to the stabilization of the gel structure (Ghorbani Gorji et al., 2018).

hydrogen bonds, or by an alginate-like mechanism of calcium bridge formation. However, the content of ether sulphate, whose proportion in the molecule of 1carrageenan is up to $32 \%$, prevents the formation of multilayered, even double helices, thereby reducing the strength of the gel. Therefore, 1 -carrageenan gels have a soft, elastic structure and are less susceptible to degradation as compared to sodium alginate (Hotchkiss et al., 2016). The structure of the gelatin containing cream also undergoes delamination with the formation of non-uniform systems and partial separation of the liquid. However, the final viscosity (of the practically destroyed structure) of the 
creams with gelatin was 10-15 times higher than that of polysaccharides. An explanation may be the gelation process of gelatin caused by the conformational rearrangement of individual polypeptide chains into an ordered spiral-type network, as well as the intramolecular interactions of gelatin with milk casein and the formation of a "protein-protein" system (Harikedua, 1997). The texture of such gels will depend on the concentration of gelatin and the $\mathrm{pH}$ of the medium (Luo et al., 2019). Thus, the emulsion-foam system of the cream with sodium alginate can be recommended for the formation of decorations for cakes and other structures. This applies to creams, both immediately after manufacture and some time after preparation in low temperature conditions.

Table2. Rheological parameters of the cream systems under study $(n=3, p \leq 0.05)$.

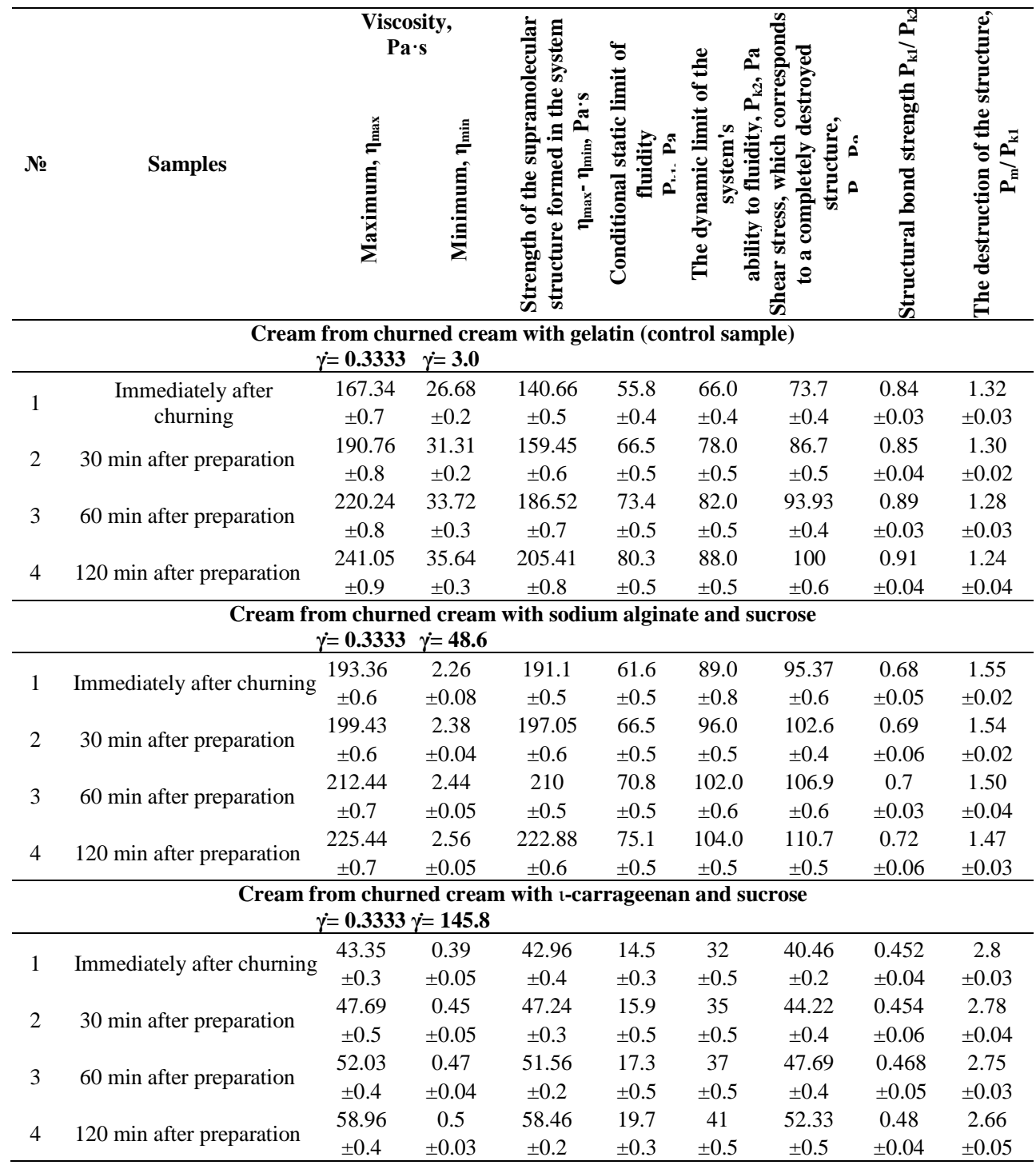


1-carrageenan can form an associative gel network, either as a result of numerous

Also, we can conclude that the prepared 1-carrageenan creams should not be subjected to intense further mixing, using whipping machines (for example, at a speed of the working body of the whipping mechanism of $300 \mathrm{rpm}$ ), as the system loses its integral structure. Such emulsion-foam systems of creams can be recommended for interlacing semi-finished baked products, filling the cavities of cakes and eclairs, etc. Studies of the springy-plastic characteristics of the emulsionfoam systems of the churned cream were carried out immediately after preparation (0 min), then after 30,60 and 120 minutes following the formation dynamics of the structure development. The deformation curves of the emulsion-foam structure of the creams under study are seen in Figures 7-9.

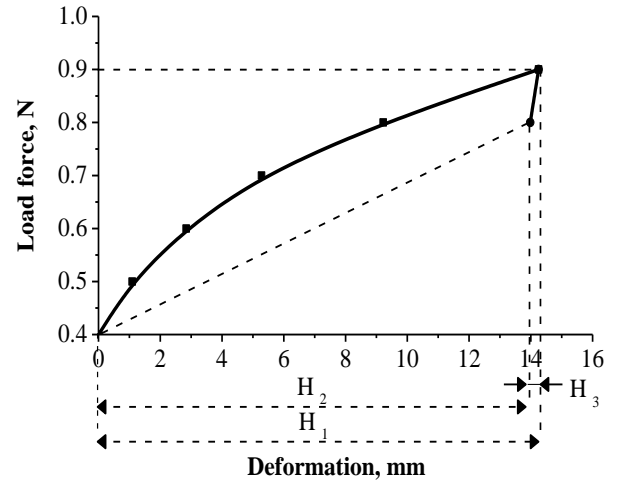

a) immediately after churning

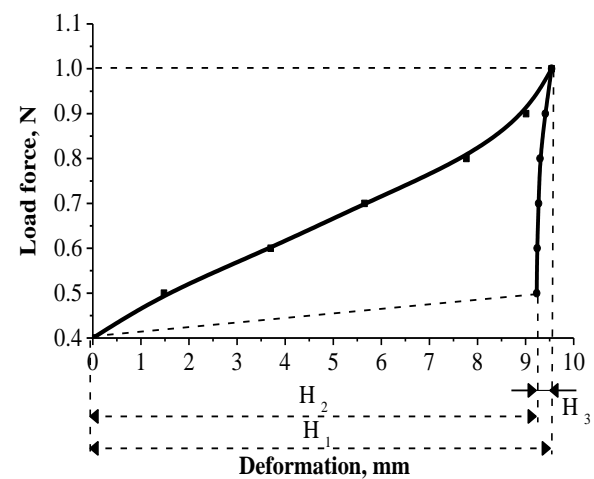

c) 60 min after preparation

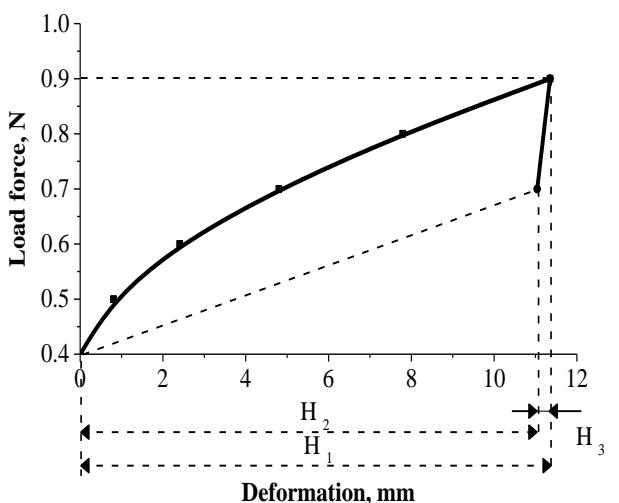

b) 30 min after preparation

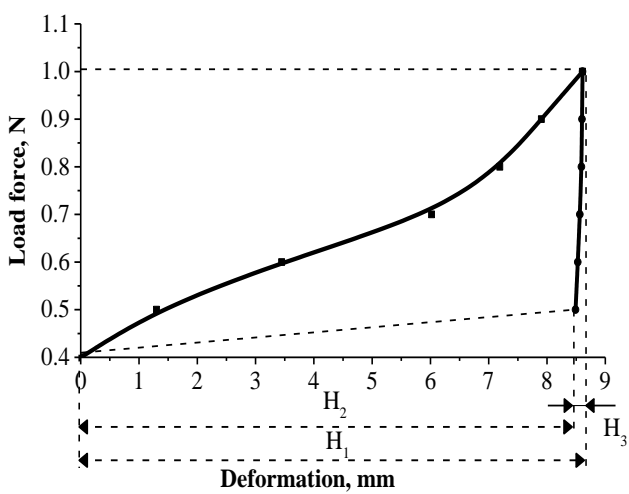

d) 120 min after preparation

Figure 7. Curves of structural-mechanical quality indicators of the emulsion-foam cream system with gelatin.

The appearance of the curves clearly confirmed that the structure of the obtained creams changed over time. For all the samples investigated, 2 hours after preparation the resisting curves changed their orientation, the area under the curve (S) increased, while the registered deformation decreased. For the samples with gelatin and sodium alginate, the load force used for the measurements increased after $60 \mathrm{~min}$. A detailed analysis of the curves is included in Table 3. 


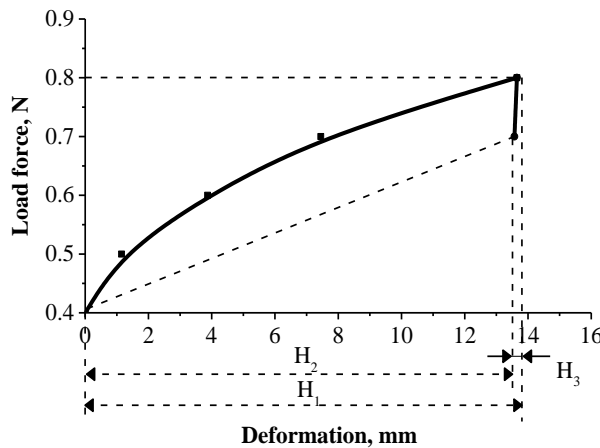

a) immediately after churning

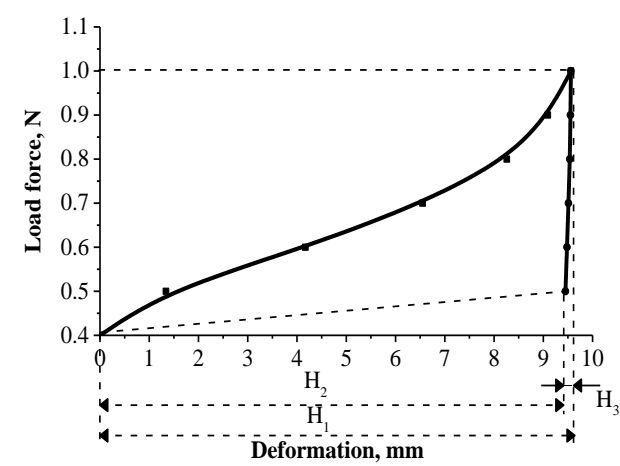

c) 60 min after preparation

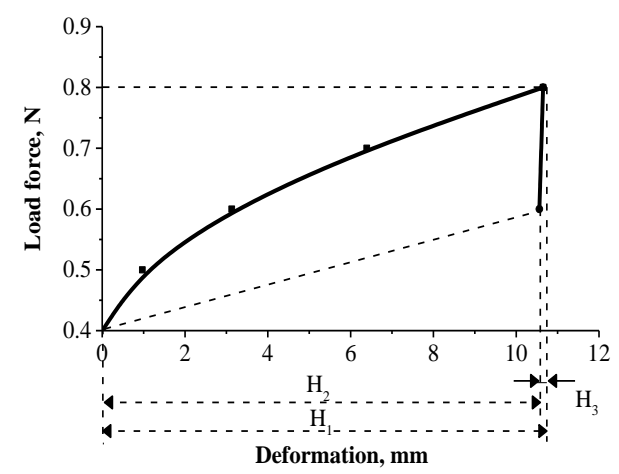

b) 30 min after preparation

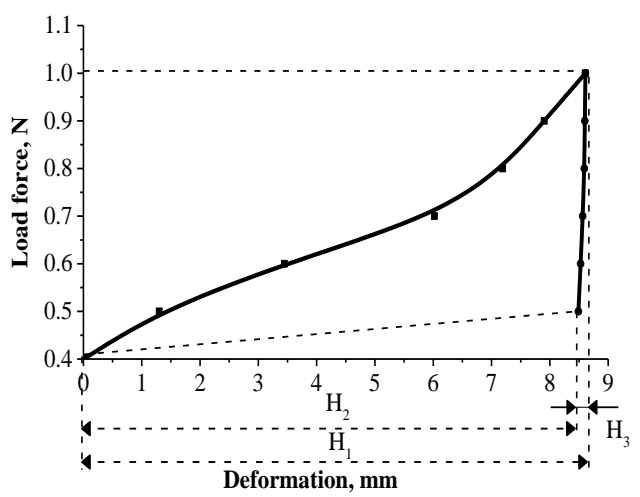

d) 120 min after preparation

Figure 8. Curves of structural-mechanical quality indicators of the emulsion-foam cream system with sodium alginate.

The table data numerically prove that the total deformation of the emulsion-foam structure of all the samples decreased with time, which is a confirmation of the development of the gel frame of the structure-forming agents. That is, the structure of the creams some time after preparation becomes stable. This is a positive factor that proves the feasibility of hydrocolloid introduction to ensure the stability of the emulsion-foam system. At the same time, the highest values of the total deformation were recorded for the structures with 1-carrageenan: depending on the time after preparation, the index of total deformation was $0.01953 \ldots 0.01647$. This is consistent with the viscosity for 1-carrageenan creams, which are marked as the lowest among polysaccharides.

For all the cream samples tested, the conditional strength limit or the nominal maximum stress occurring under the action of loading, at which the maximum uniform deformation over the entire length of the sample decreased over time, i.e. within systems, the stress caused by the deformation of the sample decreased. This is naturally due to the reduction of the total deformation of the samples and indicates the overall system structuring. 


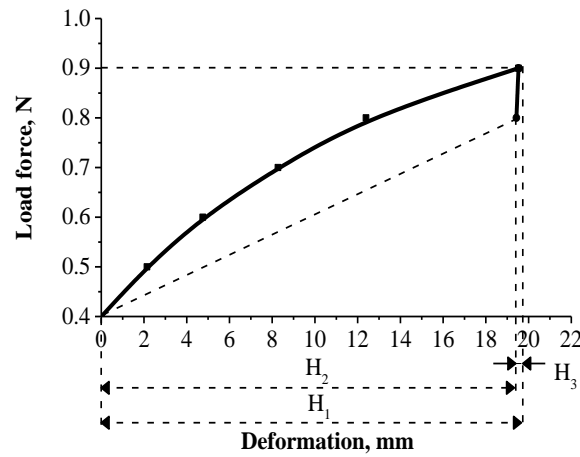

a) immediately after churning

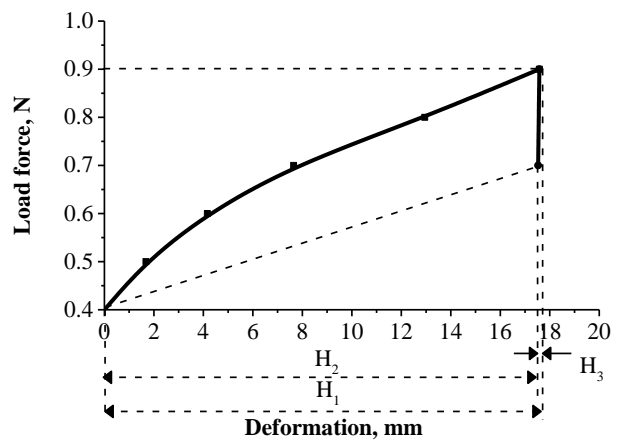

c) 60 min after preparation

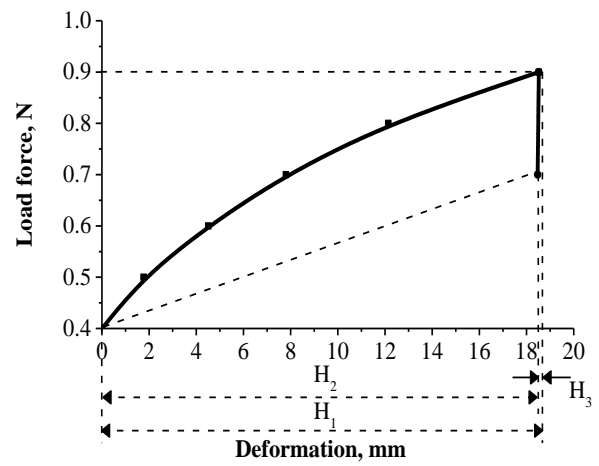

b) 30 min after preparation

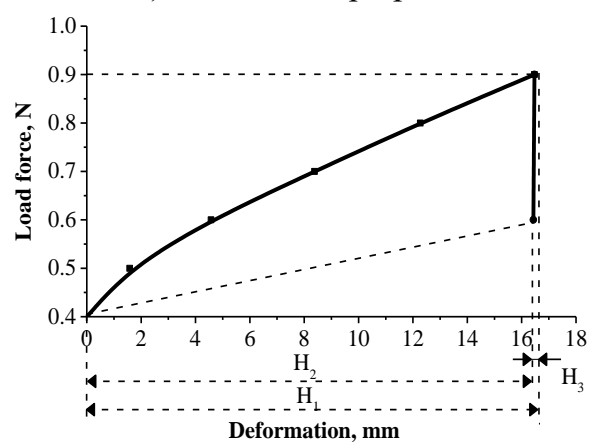

d) 120 min after preparation

Figure 9. Curves of structural-mechanical quality indicators of the emulsion-foam cream system with i-carrageenan.

For all the samples, the development of plastic deformation exceeded by far the development of springy deformation. In the total deformation, the proportion of plastic deformation was $98-99 \%$, and the rest was springy deformation. Some time after preparation, the ratio changed: the springy fraction increased for the samples with gelatin and sodium alginate, and decreased for the samples with 1carrageenan. For example, the relative springy deformation for the samples with gelatin was $1.89 \ldots 7.31 \%$, for the samples with sodium alginate $-0.59 \ldots 1.39 \%$, and for the samples with 1 -carrageenan $-0.51 \ldots 0.24 \%$. This indicates that plasticity developed in the samples of gel systems with 1-carrageenan, and springiness developed in the samples with gelatin or sodium alginate. As compared to the control sample with gelatin, the creams with both polysaccharides were characterized by greater plasticity.

These characteristics suggest that creams with polysaccharides will have more time to be used to decorate different confectionery than the creams with gelatin. Some time after preparation, they will have sufficient plasticity to be used to decorate cakes and to fill semi-finished baked products. 
Table 3. Structural-mechanical quality indicators of the emulsion-foam cream systems

\begin{tabular}{|c|c|c|c|c|}
\hline \multirow{2}{*}{ Indicators } & \multicolumn{4}{|c|}{ Time, min } \\
\hline & $\mathbf{0}$ & 30 & 60 & 120 \\
\hline \multicolumn{5}{|c|}{ Emulsion-foam system of cream with gelatin } \\
\hline \multirow{2}{*}{ Total deformation, m } & 0.01425 & 0.01135 & 0.00954 & 0.00520 \\
\hline & $\pm 4 \cdot 10^{-3}$ & $\pm 3 \cdot 10^{-3}$ & $\pm 5 \cdot 10^{-4}$ & $\pm 3 \cdot 10^{-4}$ \\
\hline \multirow{2}{*}{ Plastic deformation, $\mathrm{m}$} & 0.01398 & 0.01104 & 0.00922 & 0.00482 \\
\hline & $\pm 5 \cdot 10^{-3}$ & $\pm 4 \cdot 10^{-3}$ & $\pm 3 \cdot 10^{-4}$ & $\pm 3 \cdot 10^{-4}$ \\
\hline Relative plastic deformation, $\%$ & $98.11 \pm 0.4$ & $97.27 \pm 0.5$ & $96.65 \pm 0.4$ & $92.69 \pm 0.4$ \\
\hline Relative springy deformation, $\%$ & $1.89 \pm 0.08$ & $2.73 \pm 0.06$ & $3.35 \pm 0.04$ & $7.31 \pm 0.03$ \\
\hline \multirow{2}{*}{ Conditional strength limit, $\mathrm{Pa}$} & 8527.23 & 8319.25 & 7695.31 & 7383.34 \\
\hline & \pm 45.0 & \pm 34.0 & \pm 45.0 & \pm 40.0 \\
\hline Specific work of plastic deformation, $\mathrm{J} / \mathrm{m}^{3}$ & $3.71 \pm 0.07$ & $2.83 \pm 0.05$ & $2.77 \pm 0.05$ & $1.09 \pm 0.08$ \\
\hline \multicolumn{5}{|c|}{ Emulsion-foam system of cream with sodium alginate } \\
\hline \multirow{2}{*}{ Total deformation, $\mathrm{m}$} & 0.01365 & 0.01054 & 0.00956 & 0.00861 \\
\hline & $\pm 5 \cdot 10^{-3}$ & $\pm 6 \cdot 10^{-3}$ & $\pm 4 \cdot 10^{-4}$ & $\pm 3 \cdot 10^{-4}$ \\
\hline \multirow{2}{*}{ Plastic deformation, $\mathrm{m}$} & 0.01357 & 0.01045 & 0.00947 & 0.00849 \\
\hline & $\pm 5 \cdot 10^{-3}$ & $\pm 4 \cdot 10^{-3}$ & $\pm 7 \cdot 10^{-4}$ & $\pm 5 \cdot 10^{-4}$ \\
\hline Relative plastic deformation, $\%$ & $99.41 \pm 0.4$ & $99.15 \pm 0.3$ & $99.06 \pm 0.3$ & $98.61 \pm 0.3$ \\
\hline Relative springy deformation, $\%$ & $0.59 \pm 0.08$ & $0.85 \pm 0.07$ & $0.94 \pm 0.09$ & $1.39 \pm 0.09$ \\
\hline \multirow{2}{*}{ Conditional strength limit, $\mathrm{Pa}$} & 7695.31 & 7591.32 & 7175.35 & 6967.37 \\
\hline & \pm 38.0 & \pm 34.0 & \pm 35.0 & \pm 31.0 \\
\hline Specific work of plastic deformation, $\mathrm{J} / \mathrm{m}^{3}$ & $2.95 \pm 0.05$ & $2.38 \pm 0.05$ & $2.33 \pm 0.05$ & $2.21 \pm 0.05$ \\
\hline \multicolumn{5}{|c|}{ Emulsion-foam system of cream with l-carrageenan } \\
\hline \multirow{2}{*}{ Total deformation, $\mathrm{m}$} & 0.01953 & 0.01852 & 0.01758 & 0.01647 \\
\hline & $\pm 5 \cdot 10^{-3}$ & $\pm 5 \cdot 10^{-3}$ & $\pm 4 \cdot 10^{-4}$ & $\pm 3 \cdot 10^{-4}$ \\
\hline \multirow{2}{*}{ Plastic deformation, $\mathrm{m}$} & 0.01943 & 0.01846 & 0.01753 & 0.01643 \\
\hline & $\pm 5 \cdot 10^{-3}$ & $\pm 6 \cdot 10^{-3}$ & $\pm 6 \cdot 10^{-3}$ & $\pm 4 \cdot 10^{-3}$ \\
\hline Relative plastic deformation, $\%$ & $99.46 \pm 0.5$ & $99.68 \pm 0.5$ & $99.72 \pm 0.5$ & $99.76 \pm 0.5$ \\
\hline Relative springy deformation, $\%$ & $0.51 \pm 0.06$ & $0.32 \pm 0.04$ & $0.28 \pm 0.04$ & $0.24 \pm 0.04$ \\
\hline \multirow{2}{*}{ Conditional strength limit, $\mathrm{Pa}$} & 8319.25 & 7799.30 & 7591.32 & 7383.34 \\
\hline & \pm 38.0 & \pm 35.0 & \pm 30.0 & \pm 36.0 \\
\hline Specific work of plastic deformation, $\mathrm{J} / \mathrm{m}^{3}$ & $5.87 \pm 0.05$ & $5.38 \pm 0.03$ & $5.01 \pm 0.03$ & $4.90 \pm 0.03$ \\
\hline
\end{tabular}

The creams with gelatin develop a sufficiently resilient structure that can be irreversibly destroyed during the test. This makes it impossible to use them for decoration shortly after preparation.

For all the samples, including the sample with gelatin, the specific work of plastic deformation decreased, characterizing the energy consumed by the sample for deformation processes. For example, the specific work of plastic deformation for the samples with gelatin was $3.71-1.09 \mathrm{~J} / \mathrm{m}^{3}$, for the samples with sodium alginate 2.95-2.21 J/m $\mathrm{m}^{3}$, and for the samples with $\mathrm{t}$-carrageenan $-5.87-4.90 \mathrm{~J} / \mathrm{m}^{3}$. The specific work of plastic deformation was in direct correlation with the total deformation and, accordingly, its reduction reduced the following indicator.

Springy deformation changes were more pronounced in the creams with gelatin, which indicates the development of the most elastic gel framework among all the experimental systems. It showed the highest strength among all the samples - the total deformation was reduced by $64 \%$ (from 0.01425 to $0.00520 \mathrm{~m}$ ) as compared 
to sodium alginate, the total deformation of which was reduced by $37 \%$ (from 0.01365 to $0.00861 \mathrm{~m}$ ). That is, the structure of the creams with t-carrageenan were characterized as the most stable when subjected to mechanical loading.

The introduction of different types of sugars changes the structural and mechanical properties, such as springiness and plasticity, resistance to deformation, which requires the determination of the state of the water forms of the dispersion medium in the creams.

\section{Conclusions}

The present study focuses on the feasibility of using sodium alginate, t-carrageenan and gelatin to stabilize emulsion-foam systems in churned cream. Such systems may form the basis for low energy value creams.

It was determined that the emulsion-foam structures of the cream samples studied belong to the category of pseudoplastic non-Newtonian fluids. Different values of the conditional dynamic limit of fluidity were determined: for sodium alginate $100 \mathrm{~Pa}$ and more, for 1 -carrageenan $\sim 35 \mathrm{~Pa}$, for gelatin - $66 \mathrm{~Pa}$ and more, which is connected to a high level of swelling of the hydrocolloids in the creams and their differences in structure-forming manners.

Two hours after preparation, all the samples were characterized by a decrease in the total deformation. These actions point out the general structuring of the system and ensure the feasibility of the introduction of hydrocolloids to obtain stable emulsion-foam systems.

For the samples studied, the development of plastic deformations exceeded that of springy deformations. In the structure of the experimental samples, plastic deformations exceed springy ones: the relative plastic deformation of the samples of the emulsion-foam systems after churning was $98 \ldots 99 \%$. Over time a part of the springy deformation increased for the samples with gelatin and sodium alginate, and decreased for the samples with i-carrageenan.

The emulsion-foam system of the cream with sodium alginate can be recommended for use in decorating cakes etc. This applies to creams, both immediately after manufacture and some time after preparation, in low temperature conditions.

The creams prepared with t-carrageenan should not be subjected to intense further churning, using whipping machines (for example, at a frequency of the working body of the whipping mechanism of $300 \mathrm{rpm}$ ), during which the system loses its integral structure. Such emulsion-foam systems of creams can be recommended for interlacing semi-finished baked products, filling the cavities of cakes and eclairs, etc.

The emulsion-foam systems of the creams with gelatin developed more of a springy structure some time after preparation when compared to the creams with polysaccharides. Such systems are subject to irreversible deformation under the influence of mechanical movement, so it is recommended not to use them to decorate products some time after preparation; they should be used immediately. 


\section{References}

Al-Amin, Esraa Gaffar, A. 2019. Production and Evaluation of Some Quality Attributes of Goat Milk Ice Cream. Sudan.

Allen, C. Buhler, O., Arthur, P. Hansen, S. 2005. Low-calorie low-fat butter-flavored topping compositions and methods of preparation, Patent US 7368143 United States: Patent Application Publication.

Barata, M., Guillemant, M., Moretti, E., Muller, E., Delebarre, M. 2019. Patent of the USA US20190045826 «Nutritional formulations such as a yoghurt, cream, cream dessert or frozen dessert, comprising a pea protein isolate, and the use of the formulation as a source of protein».

Camacho, M., Martinez-Navarrete, N., Chiralt, A. 2005. Rheological characterization of experimental dairy creams formulated with locust bean gum (LBG) and $\lambda$-carrageenan combinations. International Dairy Journal, 15, 243-248.

Chen, Y., He, Q., Shi, X., Wang, T., Zhang, B. 2016. Unsteady flow of shear-thinning nonNewtonian incompressible fluid through a twin-screw extruder. Acta Mathematicae Applicatae Sinica, English Series, 32, 423-436.

Dewettinck, K., Rombaut, R., Thienpont, N., Le, T.T., Messens, K. Van Camp, J. 2008. Nutritional and technological aspects of milk fat globule membrane material. International Dairy Journal, 18, 436-457.

Duan, R., Zhang, J., Liu, L., Cui, W., Regenstein, J.M. 2018. The functional properties and application of gelatin derived from the skin of channel catfish (ictalurus punctatus). Food chemistry, 239, 464-469.

Erkebaev, M., Kulazhanov, T., Medvedkov, E. 2006. Food products rheology basis. Almaty.

Ershova, T., Levochkina, L., Bozhko, S. 2008. New functional creams. Confectionery production, 2,14 .

Farahmandfar, R., Asnaashari, M., Salahi, M.R., Khosravi Rad, T. 2017. Effects of basil seed gum, cress seed gum and quince seed gum on the physical, textural and rheological properties of whipped cream. International Journal of Biological Macromolecules, 98, 820-828.

Farahmandfar, R., Asnaashari, M., Taheri, A., Rad, T.K. 2019. Flow behavior, viscoelastic, textural and foaming characterization of whipped cream: Influence of Lallemantia royleana seed, Salvia macrosiphon seed and carrageenan gums. International Journal of Biological Macromolecules, 121, 609-615.

Ghorbani Gorji, E.G., Waheed, A., Ludwig,R., Toca-Herrera,J.L., Schleining,G., Ghorbani Gorji,S.G. 2018. Complex Coacervation of Milk Proteins with Sodium Alginate.J Agric Food Chemistry, 66, 3210-3220.

Goff, H.D., Spagnuolo, P. 2001. Effect of stabilizers on fat destabilization measurements in ice cream. Milchwissenschaft, 56, 450-453.

Gurov, A. 2007. Patent of Russia 2294111 «The method of production of confectionery creamchurned mass and confectionery cream-churned mass obtained by this method», Moscow: State Patent Office of Russia.

Harikedua, S.D. 2019. Protein gels: a mini review. Jurnal Media Teknologi Hasil Perikanan, 7, 19-24. 
Hartel, R. W., Joachim, H., Hofberger, R. 2018. Confectionery science and technology. Springer international publishing.

Hotchkiss, S., Brooks, M., Campbell, R., Philp, K., Trius, A. 2016. The use of carrageenan in food. In.: Pereira I. Carrageenans: sources and extraction methods, molecular structure, bioactive properties and health effects, $1^{\text {st }}$ edn. Nova Science Publications Inc., New York.

Javidi, F., Razavi, S. 2019. New Hydrocolloids in Ice Cream.Emerging Natural Hydrocolloids: Rheology and Functions, 21.

Jiao, W., Chen, W., Mei, Y., Yun, Y., Wang, B., Zhong, Q., Chen, H., Chen, W. 2019. Effects of Molecular Weight and Guluronic Acid/Mannuronic Acid Ratio on the Rheological Behavior and Stabilizing Property of Sodium Alginate. Molecules, 24, $43-$ 74.

Kaledina, V., Fedosova, N. 2017. New approaches to creating functional products for a closed milk-polysaccharide system. Foods and Raw Materials, 5, 44-53.

Kambulova, Y., Zviahintseva-Semenets, Y., Kobilinska, O., Yatsenko, V. 2017. Rheological properties of low-fat creams with polysaccharides and sugars. The Agrofood Industry, 6, 24-28.

King, A.2019. Brown seaweed extracts (alginates). Food Hydrocolloids, 2, 115- 191.

Kovalevska, E. 2010. Rheology of food masses. NUFT. Kyiv.

Levit, I., Sukmanov, V., Afenchenko D. 2014. Rheology of food products. Donetsk

Lingerud, S. 2009. Churned non-dairy cream with low fat content. Patent of Russia 2346543, Moscow: State Patent Office of Russia.

Luo, Y., Liu, X., Pang, Z. 2019. Tribo-rheological properties of acid milk gels with different types of gelatin: Effect of concentration. Journal of Dairy Science, 102, 78497862.

Lysyuk, G., Samokhvalova, O., Kucheruk, Z., Postnova, O., Oliynyk, S., Artamonova, M., Nemirich, O., Starchayenko, O. 2007. Technology of confectionery and bakery products, Kharkiv.

Melnychuk, O., Kambulova, Y, Kropyvnytska, I., Obolkina, V., Osipenko, U., Antonyuk, M. 2011. Method of producing cream. Patent of Ukraine 56647, Kyiv. State Patent Office of Ukraine.

Mezger, T. G. 2006. The rheology handbook: for users of rotational and oscillatory rheometers. Vincentz Network GmbH \& Co KG.

Milliatti, M.C., Lannes, S. 2018. Impact of stabilizers on the rheological properties of ice creams. Food Science and Technology, 38, 793-799.

Mureșan, V., Danthine, S., Petrut, R.F., Racolța, E., Muste, S., Mureșan, A.E., Socaciu, C., Blecker, C. 2018. Enhancing the Unsaturated Fat Content and Techno-Functional Properties of Whipped Creams by Designing New Structured Fats - Blends of Rice Bran Wax Oleogel and Butter as Fat Model Systems. Technology and Nutrition in a Changing World.

Nguyen, V., Duong, C.T.M., Vu, V. 2015. Effect of thermal treatment on physical properties and stability of whipping and whipped cream. Journal of Food Engineering, $163,32-36$.

Pang, Z., Deeth, H., Prakash, S., Bansal, N. 2016. Development of rheological and sensory properties of combinations of milk proteins and gelling polysaccharides as potential 
gelatin replacements in the manufacture of stirred acid milk gels and yogurt. Journal of Food Engineering, 169, 27-37.

Radziyevska, I., Komarenko, Y. 2013. Some aspects of the combination of the composition of low-calorie emulsion cream. Products \& Ingredients, 8, 12-13.

Rahimah, S., Fadhila, A., Lembong, E., Sukri, N., Cahyanto, T. 2020. Characteristics of spanish mackerel (Scomberomorus commerson) bone gelatin for ice cream stabilizer. Indonesian journal of halal research, 2, 1-7.

Romanova, E., Kudinova, V. 2008. Stabilization of properties of churned finishing semi-finished products based on vegetable cream. Bread products, 2, 64-65.

Schamm, L. 2006. Emulsions, Foams, and Suspensions: Fundamentals and Applications, Germany.

Schmidt, G.M., Kimmel, J.L., Handrick, A.S., Dierbach, L.A., Mikeska, K.A., Campbell, B.E., Robinson, K. 2016. Heat stable concentrated dairy liquid and cream product. Patent of the USA US20100055290A1.

Srikaeo, K., Doungjan, S. 2020. Hydrocolloids retard lipid digestion in whipping cream. Food Research, 4, 831 - 838.

UrRehman, K., Khan, A., Malika, M.Y., Pradhanb, R.K. 2017. Combined effects of Joule heating and chemical reaction on non-Newtonian fluid in double stratified medium: A numerical study. Results in Physics, 7, 3487-3496.

Volovik, L., Kovalevska, E., Mank, V. 1999. Colloidal chemistry. NUFT. Kyiv.

Yousefia, M., Jafari, S.M. 2019. Recent advances in application of different hydrocolloids in dairy products to improve their techno-functional properties. Trends in Food Science \& Technology, 88, 468-483.

Zaouadi, N., Ziane-Zafour, A.H., Ouazib, M., Arab, Y., Hacini, K., Aslan, S.S. 2019. Formulation and Optimization by Experimental Design of Dairy Dessert Based on Lupinus albus L. and Stevia rebaudiana Extracts. Asian Journal of Dairy \& Food Research, 38, 281-287.

Zviahintseva-Semenets, Y., Kambulova, Y., Sokolovska, I., Kobilinska, O., Kolesnik, M. 2016. Investigation of the process of swelling of polysaccharides for use in the technology of creams. Food Science and Technology, 10, 24-31. 